\title{
A VARIATIONAL INEQUALITY ASSOCIATED WITH A LUBRICATION PROBLEM
}

\author{
By
}

\section{Jong-Shenq Guo}

IMA Preprint Series \# 530

$$
\text { June } 1989
$$




\title{
A VARIATIONAL INEQUALITY ASSOCIATED WITH A LUBRICATION PROBLEM
}

\author{
Jong-Shenq Guo \\ School of Mathematics \\ University of Minnesota \\ Minneapolis, Minnesota 55455
}

Key words and phrases: Variational inequality, lubrication problem, free boundary, Reynolds variational inequality.

§0. Introduction. In this paper, we study a variational inequality which describes a lubrication problem in a journal bearing. For the physical background, we refer the reader to Bayada[3] and Bayada and Chambat[4] for details. Given a constant $a \in(0,1)$ and a smooth function $\theta(y)$ defined in $[0,1]$ such that

$$
(1-a) /(1+a)<\theta(y)<1, \forall y \in[0,1]
$$

let $h(x)=1+a \cos x, g(x, y)=h(x)-(1+a) \theta(y)$, and introduce the sets(see Figure 1)

$$
\begin{aligned}
& \Omega=(0,2 \pi) \times(0,1), \quad R_{1}=(0, \pi) \times(0,1), \quad R_{2}=(\pi, 2 \pi) \times(0,1), \\
& \Omega_{1}=\left\{(x, y) \in R_{1} ; g(x, y)>0\right\}, \quad \Omega_{2}=\Omega \backslash \overline{\Omega_{1}}, \quad T=\partial \Omega_{1} \cap \Omega, \\
& \partial_{0} \Omega=\{0\} \times(0,1), \quad \partial_{1} \Omega=\partial \Omega \backslash \partial_{0} \Omega .
\end{aligned}
$$

Let

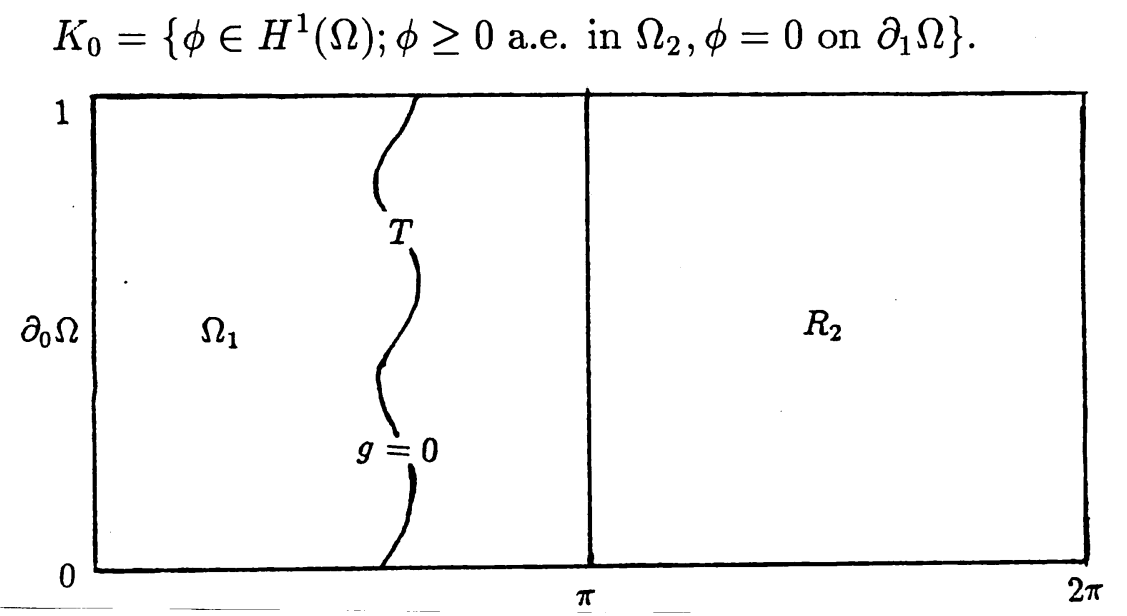

Figure 1

Note that $T \subset R_{1}$ and $g=0$ on $T$ by the assumption on $\theta$. The following classical formulation of the lubrication problem was posed in [4]. 
$\operatorname{Problem}\left(P_{c}\right)$. Find a non-negative continuous function $u(x, y)$ in $\bar{\Omega}$ and two disjoint free boundaries $S_{1}$ and $S_{2}$ such that the following conditions hold:

$$
\begin{aligned}
& S_{1} \subset \Omega_{1}, S_{2} \subset R_{2}, \\
& -\nabla \cdot\left(h^{3} \nabla u\right)=a \sin x \text { in } \Omega^{+} \equiv\{u>0\}, \\
& u=0 \quad \text { on } \partial \Omega, \\
& u=0 \quad \text { on } S \equiv \partial \Omega^{+} \cap \Omega=S_{1} \cup S_{2}, \\
& h^{3} \frac{\partial u}{\partial \nu}=g \nu_{x} \quad \text { on } S_{1}, \\
& \frac{\partial u}{\partial \nu}=0 \quad \text { on } S_{2},
\end{aligned}
$$

where $\nabla=(\partial / \partial x, \partial / \partial y), \nu$ is the unit outer normal to $\partial \Omega^{+}, \nu=\left(\nu_{x}, \nu_{y}\right)$, and $\partial u / \partial \nu$ is defined from the right-hand side of $S_{1}$ and from the left-hand side of $S_{2}$.

Hereafter $A^{+}(u)$ or $A^{+}$will denote the set $A \cap\{u>0\}$. It is anticipated that the set $\{u=0\}$ lies to the left of $S_{1}$ and to the right of $S_{2}$. To solve this problem, Bayada and Chambat [4] considered the following variational inequality which is a weak formulation of $\operatorname{Problem}\left(P_{c}\right)$.

$\operatorname{Problem}(P)$. Find $u \in K_{0}$ such that

$$
\int_{\Omega} h^{3} \nabla u \cdot \nabla(\phi-u) \geq \int_{\Omega_{1}} I_{\Omega_{1}^{+}(u)} g(\phi-u)_{x}+\int_{\Omega_{2}} g(\phi-u)_{x},
$$

for all $\phi \in K_{0}$, where $I_{A}$ denotes the characteristic function of $A$.

In this paper the subscript $x$ or $y$ will always represent the partial derivative of functions with respect to that variable. Note that we do not require functions in $K_{0}$ vanish on $\partial_{0} \Omega$. In [4], the authors proved existence of a solution to the $\epsilon$-problem(see Lemma 1.1 in $\S 1$ ). However, they have not been able to use it to establish existence of a solution to Problem $(P)$ (unless they a priori assume that the free boundary is Lipschitz). We instead start with a weaker formulation:

$\operatorname{Problem}\left(P_{0}\right)$. Find $(u, \gamma), u \in K_{0}, \gamma \in L^{\infty}\left(\Omega_{1}\right), 0 \leq \gamma \leq 1$ a.e. in $\Omega_{1}$ and $\gamma \subset H(u)$, such that

$$
\int_{\Omega} h^{3} \nabla u \cdot \nabla(\phi-u) \geq \int_{\Omega_{1}} \gamma g(\phi-u)_{x}+\int_{\Omega_{2}}(a \sin x)(\phi-u),
$$

for all $\phi \in K_{0}$, where $H(u)$ is the Heaviside graph of $u$ (i.e., $H(t)=1$ if $t>0,=0$ if $t<0$, and $=[0,1]$ if $t=0)$.

We shall establish existence of a solution to $\operatorname{Problem}\left(P_{0}\right)$ and prove a regularity theorem for solutions to $\operatorname{Problem}\left(P_{0}\right)$ in $\S 1$. In $\S 2$ we shall be using, among other methods, the 
comparison argument of [4] to prove, under some condition on $\theta$, that any solution of Problem $\left(P_{0}\right)$ vanishes on all of $\partial \Omega$; we shall also derive some properties of the free boundary and the non-coincidence set $\Omega^{+}$. In $\S 3$ we prove that $\gamma=I_{\Omega_{1}^{+}(u)}$ for any solution $(u, \gamma)$ of $\operatorname{Problem}\left(P_{0}\right)$ and establish some regularity results of the free boundary. Since $g=0$ on $T$ by assumption, the second integrals on the right-hand sides of $(0.7)$ and $(0.8)$ are equal. Therefore, the two problems $\left(P_{0}\right)$ and $(P)$ are equivalent. Finally, in $\S 4$ we shall prove that there exists only one solution to $\operatorname{Problem}\left(P_{0}\right)$.

$\S 1$. Existence and regularity. For any $\epsilon>0$, let

$$
H_{\epsilon}(t)=\left\{\begin{array}{l}
0 \text { if } t<0 \\
t / \epsilon \text { if } 0 \leq t \leq \epsilon \\
1 \text { if } t>\epsilon
\end{array}\right.
$$

Lemma 1.1. There exists a $u_{\epsilon} \in K_{0}, u_{\epsilon} \geq 0$ in $\Omega$, such that the inequality

$$
\int_{\Omega} h^{3} \nabla u_{\epsilon} \cdot \nabla\left(\phi-u_{\epsilon}\right) \geq \int_{\Omega_{1}} H_{\epsilon}\left(u_{\epsilon}\right) g\left(\phi-u_{\epsilon}\right)_{x}+\int_{\Omega_{2}}(a \sin x)\left(\phi-u_{\epsilon}\right)
$$

holds for all $\phi \in K_{0}$. Moreover,

$$
\int_{\Omega}\left|\nabla u_{\epsilon}\right|^{2} \leq C
$$

where $C$ is a positive constant independent of $\epsilon$.

The proof of Lemma 1.1 is given in [4].

Taking $\phi=u_{\epsilon} \pm \zeta$ with $\zeta \in C_{0}^{\infty}\left(\Omega_{1}\right)$ in (1.1), we deduce that $u_{\epsilon}$ is a weak solution of the equation

$$
-\nabla \cdot\left(h^{3} \nabla u_{\epsilon}\right)=-\left(H_{\epsilon}\left(u_{\epsilon}\right) g\right)_{x} \text { in } \Omega_{1} .
$$

Next, taking $\zeta \in C^{\infty}\left(\Omega_{1}\right), \zeta=0$ on $\partial \Omega_{1} \backslash \partial_{0} \Omega$, we obtain

$$
\int_{\partial_{0} \Omega} h^{3} \frac{\partial u_{\epsilon}}{\partial x} \zeta=\int_{\partial_{0} \Omega} H_{\epsilon}\left(u_{\epsilon}\right) g \zeta
$$

or equivalently,

$$
\frac{\partial u_{\epsilon}}{\partial x}=\frac{1-\theta(y)}{(1+a)^{2}} H_{\epsilon}\left(u_{\epsilon}\right) \quad \text { on } \quad \partial_{0} \Omega .
$$

From (1.3), (1.4) and $u_{\epsilon}=0$ on $\partial_{1} \Omega$ it follows by elliptic regularity theory(cf. [7] and [10]) that

$$
\left\|u_{\epsilon}\right\|_{C^{\alpha}\left(\overline{\Omega^{\prime}}\right)} \leq C, \forall \Omega^{\prime} \subset \subset \overline{\Omega_{1}} \backslash T, \forall \alpha \in(0,1),
$$

where $C$ is a positive constant independent of $\epsilon$. 
Theorem 1.2. There exists a solution $(u, \gamma)$ of $\operatorname{Problem}\left(P_{0}\right)$.

Proof. By (1.2) and (1.5), there is a sequence $\left\{\epsilon_{n}\right\}, \epsilon_{n} \downarrow 0$ as $n \rightarrow \infty$, such that $u_{\epsilon_{n}} \rightarrow u$ weakly in $H^{1}(\Omega)$,

$u_{\epsilon_{n}} \rightarrow u \quad$ uniformly on any compact subset of $\overline{\Omega_{1}} \backslash T$,

$u_{\epsilon_{n}} \rightarrow u$ strongly in $L^{2}(\Omega)$ and a.e. in $\Omega$,

$H_{\epsilon_{n}}\left(u_{\epsilon_{n}}\right) \rightarrow \gamma$ weakly in $L^{2}\left(\Omega_{1}\right)$ and weakly* in $L^{\infty}\left(\Omega_{1}\right)$.

Also, $\gamma=1$ a.e. in $\Omega_{1}^{+}$and $0 \leq \gamma \leq 1$ a.e. in $\Omega_{1}$.

For any $\phi \in K_{0}$ we have

$$
\begin{aligned}
& \int_{\Omega} h^{3} \nabla u_{\epsilon_{n}} \cdot \nabla \phi \rightarrow \int_{\Omega} h^{3} \nabla u \cdot \nabla \phi, \\
& \int_{\Omega_{2}}(a \sin x)\left(\phi-u_{\epsilon_{n}}\right) \rightarrow \int_{\Omega_{2}}(a \sin x)(\phi-u), \\
& \int_{\Omega_{1}} H_{\epsilon_{n}}\left(u_{\epsilon_{n}}\right) g \phi_{x} \rightarrow \int_{\Omega_{1}} \gamma g \phi_{x},
\end{aligned}
$$

as $n \rightarrow \infty$, and

$$
\int_{\Omega} h^{3}|\nabla u|^{2} \leq \liminf _{n \rightarrow \infty} \int_{\Omega} h^{3}\left|\nabla u_{\epsilon_{n}}\right|^{2} .
$$

Introduce the function

$$
G_{\epsilon}(t)=\left\{\begin{array}{l}
0 \text { if } t \leq 0 \\
t^{2} /(2 \epsilon) \text { if } 0 \leq t \leq \epsilon \\
t-\epsilon / 2 \text { if } t \geq \epsilon
\end{array}\right.
$$

it satisfies $G_{\epsilon}^{\prime}(t)=H_{\epsilon}(t)$. Clearly

$$
\begin{aligned}
\left|G_{\epsilon_{n}}\left(u_{\epsilon_{n}}\right)-u\right| & \leq\left|G_{\epsilon_{n}}\left(u_{\epsilon_{n}}\right)-G_{\epsilon_{n}}(u)\right|+\left|G_{\epsilon_{n}}(u)-u\right| \\
& \leq\left|u_{\epsilon_{n}}-u\right|+\left|G_{\epsilon_{n}}(u)-u\right| .
\end{aligned}
$$

Hence, using the fact that $g=0$ on $T$, we get

$$
\begin{aligned}
-\int_{\Omega_{1}} H_{\epsilon_{n}}\left(u_{\epsilon_{n}}\right) g u_{\epsilon_{n}, x} & =-\int_{\Omega_{1}} G_{\epsilon_{n}}^{\prime}\left(u_{\epsilon_{n}}\right) u_{\epsilon_{n}, x} g \\
& =-\int_{\Omega_{1}} \frac{\partial}{\partial x}\left[G_{\epsilon_{n}}\left(u_{\epsilon_{n}}\right)\right] g \\
& =\int_{\Omega_{1}} G_{\epsilon_{n}}\left(u_{\epsilon_{n}}\right) g_{x}+\int_{\partial_{0} \Omega} G_{\epsilon_{n}}\left(u_{\epsilon_{n}}\right) g \\
& \rightarrow \int_{\Omega_{1}} u g_{x}+\int_{\partial_{0} \Omega} u g \quad \text { as } n \rightarrow \infty
\end{aligned}
$$


by (1.6) and (1.11). Also,

$$
\int_{\Omega_{1}} u g_{x}+\int_{\partial_{0} \Omega} u g=-\int_{\Omega_{1}} g u_{x}=-\int_{\Omega_{1}} \gamma g u_{x},
$$

since $\gamma=1$ in $\{u>0\}$. Letting $\epsilon \rightarrow 0$ in (1.1) and using (1.7-10) and (1.12-13), we conclude that $(u, \gamma)$ is a solution of $\operatorname{Problem}\left(P_{0}\right)$.

We shall denote by $B_{r}(P)$ the ball with center at $P$ and radius $r$.

Theorem 1.3. For any solution $(u, \gamma)$ of $\operatorname{Problem}\left(P_{0}\right), u \in C^{\alpha}(\bar{\Omega})$ for all $\alpha \in(0,1)$ and $u$ is real analytic in $\Omega^{+}$.

Proof. Let $(u, \gamma)$ be a solution of $\operatorname{Problem}\left(P_{0}\right)$. Substituting $\phi=u^{+} \equiv \max (u, 0)$ in $(0.8)$, we obtain that $u \geq 0$ in $\Omega$. Let

$$
K=\left\{\phi \in K_{0} ; \phi \geq 0 \text { a.e. in } \Omega\right\} .
$$

Then $(u, \gamma)$ satisfies the variational inequality $(0.8)$ for all $\phi \in K$. Since $\tilde{g} \equiv \gamma g I_{\Omega_{1}}$ and $\tilde{h} \equiv a \sin x I_{\Omega_{2}}$ are functions in $L^{\infty}(\Omega)$, it follows from Theorem 2.9 in [9] that

$$
u \in C^{\alpha}(\bar{\Omega}), \forall \alpha \in(0,1) .
$$

Note that $u$ satisfies the equation

$$
-\nabla \cdot\left(h^{3} \nabla u\right)=a \sin x \quad \text { in } \quad \Omega_{1}^{+} \cup \Omega_{2}^{+} .
$$

Let $P \in T$ be such that $u(P)>0$. Then there is a positive number $r_{0}$ such that $u>0$ in $B=B_{r_{0}}(P)$. For any $\zeta \in C_{0}^{\infty}(B)$, the function $\phi=u \pm \epsilon \zeta$ is in $K_{0}$ for any small positive $\epsilon$. By $(0.8)$

$$
\int_{B} h^{3} \nabla u \cdot \nabla \zeta=\int_{\Omega_{1} \cap B} g \zeta_{x}+\int_{\Omega_{2} \cap B}(a \sin x) \zeta=\int_{B}(a \sin x) \zeta .
$$

It follows that $u$ satisfies (1.14) also in $B$; and consequently,

$$
-\nabla \cdot\left(h^{3} \nabla u\right)=a \sin x \quad \text { in } \Omega^{+}
$$

and by elliptic regularity theory $u$ is analytic in $\Omega^{+}$.

§2. Preliminaries. Let $(u, \gamma)$ be a solution of $\operatorname{Problem}\left(P_{0}\right)$. We shall denote the free boundary by $S=S(u, \gamma)$, i.e., $S=\partial \Omega^{+} \cap \Omega$. Let

$$
\tilde{K}=\left\{\phi \in H_{0}^{1}(\Omega) ; \phi \geq 0 \text { a.e. in } \Omega\right\} .
$$

We recall the classical Reynolds variational inequality: 
$\operatorname{Problem}(\tilde{P})$. Find $\tilde{u} \in \tilde{K}$ such that

$$
\int_{\Omega} h^{3} \nabla \tilde{u} \cdot \nabla(\phi-\tilde{u}) \geq \int_{\Omega}(a \sin x)(\phi-\tilde{u}), \forall \phi \in \tilde{K} .
$$

It is well-known that there exists a unique solution $\tilde{u}$ of $\operatorname{Problem}(\tilde{P})$ such that $\tilde{u} \in$ $W^{2, p}(\Omega) \cap W_{\text {loc }}^{2, \infty}(\Omega)$ for any $1<p<\infty$. Let $\tilde{\Omega}=\{\tilde{u}>0\}$ and $\tilde{S}=\partial \tilde{\Omega} \cap \Omega$. The following lemma shows that the free boundary $\tilde{S}$ is not empty.

Lemma 2.1. There is no positive solution for the Dirichlet problem

$$
\begin{aligned}
-\nabla \cdot\left(h^{3} \nabla u\right) & =a \sin x \quad \text { in } \quad \Omega, \\
u & =0 \quad \text { on } \quad \partial \Omega .
\end{aligned}
$$

Proof. Suppose that there exists a solution $u$ of (D) such that $u>0$ in $\Omega$. Integrating the partial differential equation over $\Omega$ and applying the divergence theorem, we obtain

$$
-\int_{\partial \Omega} h^{3} \frac{\partial u}{\partial \nu}=\int_{\Omega} a \sin x=0 .
$$

Since $u>0$ in $\Omega$ and $u=0$ on $\partial \Omega$, we have

$$
\frac{\partial u}{\partial \nu} \leq 0 \quad \text { on } \quad \partial \Omega
$$

and, by the strong maximum principle,

$$
\frac{\partial u}{\partial \nu}<0 \text { on } \partial \Omega \cap \partial R_{1},
$$

since $-\nabla \cdot\left(h^{3} \nabla u\right) \geq 0$ in $R_{1}$. It follows that the left-hand side of $(2.2)$ is positive, a contradiction.

Theorem 2.2. The solution $\tilde{u}$ of $\operatorname{Problem}(\tilde{P})$ is symmetric with respect to $y=1 / 2$ and satisfies

$$
\tilde{u}_{y} \leq 0 \quad \text { in } \quad Q \equiv(0,2 \pi) \times(1 / 2,1) .
$$

The free boundary $\tilde{S}$ is non-empty and is contained in $R_{2}$. Consequently, $\tilde{S} \cap Q$ is an $\mathrm{x}$-graph which is given by

$$
y=\tilde{\rho}(x)=\sup \{\eta \in(1 / 2,1) ; \tilde{u}(x, \eta)>0\}
$$

for $x \in(\pi, 2 \pi)$ such that $u(x, 1 / 2)>0$, where $\tilde{\rho}$ is real analytic in $\{x ; \tilde{\rho}(x)<1\}$. Moreover, $\tilde{u}\left(x_{0}, 1 / 2\right)=0$ for some $x_{0} \in(\pi, 2 \pi)$ implies that $\tilde{u} \equiv 0$ in $\left[x_{0}, 2 \pi\right] \times[0,1]$, i.e., $\tilde{S} \cap\{y=1 / 2\}$ is a single point.

Proof. The first part of the theorem can be proved by using a reflection argument with the aid of the maximum principle. Utilizing a method used in [8](see also the proof of 
Theorem 3.3 below), one can obtain the Lipschitz continuity of $\tilde{\rho}$. Then from the regularity results for the free boundary of variational inequality (cf. [6, Chap.2]) the analyticity of $\tilde{\rho}$ follows. Finally, the last statement of the theorem follows from the maximum principle.

Now, we introduce the function

$$
\sigma(x)=\int_{0}^{x} \frac{a(1+\cos \xi)}{h^{3}(\xi)} d \xi .
$$

Note that $\sigma(0)=0, \sigma(x)>0$ if $x>0$, and $\sigma$ satisfies the equation

$$
-\nabla \cdot\left(h^{3} \nabla \sigma\right)=a \sin x .
$$

By the strong maximum principle

$$
\tilde{u}<\sigma \quad \text { in } \quad \Omega, \quad \frac{\partial \tilde{u}}{\partial x}<\frac{\partial \sigma}{\partial x}=\frac{2 a}{(1+a)^{3}} \quad \text { on } \partial_{0} \Omega,
$$

since $\tilde{u}=\sigma=0$ on $\partial_{0} \Omega$ and $-\nabla \cdot\left(h^{3} \nabla(\tilde{u}-\sigma)\right)=0$ in $\tilde{\Omega}$. We define a function $\tilde{\theta}(y)$ on $(0,1)$ by

$$
\frac{\partial \tilde{u}}{\partial x}(0, y)=\frac{1-\tilde{\theta}(y)}{(1+a)^{2}} .
$$

Then from (2.6) and (2.7) it follows that $\tilde{\theta}$ satisfies the condition

$$
\tilde{\theta}>\frac{1-a}{1+a} \text {. }
$$

Let

$$
\tilde{g}(x, y)=h(x)-(1+a) \tilde{\theta}(y) .
$$

We claim that $\tilde{u}$ satisfies the variational inequality:

$$
\int_{\Omega} h^{3} \nabla \tilde{u} \cdot \nabla(\phi-\tilde{u}) \geq \int_{\Omega} \tilde{g}(\phi-\tilde{u})_{x}, \forall \phi \in K_{0} .
$$

Indeed, since $\tilde{S}$ is smooth, for any $\phi \in K_{0}$, we have

$$
\begin{aligned}
\int_{\Omega} h^{3} \nabla \tilde{u} \cdot \nabla(\phi-\tilde{u}) & =\int_{\tilde{\Omega}} h^{3} \nabla \tilde{u} \cdot \nabla(\phi-\tilde{u}) \\
& =\int_{\tilde{\Omega}}(a \sin x)(\phi-\tilde{u})-\int_{\partial_{0} \Omega}(1+a)^{3} \frac{\partial \tilde{u}}{\partial x} \phi .
\end{aligned}
$$

On the other hand, the right-hand side of (2.10) can be computed as

$$
\begin{aligned}
\int_{\Omega} \tilde{g}(\phi-\tilde{u})_{x} & =\int_{\Omega}(a \sin x)(\phi-\tilde{u})-\int_{\partial_{0} \Omega}(1+a)(1-\tilde{\theta}) \phi \\
& =\int_{\tilde{\Omega}}(a \sin x)(\phi-\tilde{u})+\int_{\Omega \backslash \tilde{\Omega}}(a \sin x) \phi-\int_{\partial_{0} \Omega}(1+a)(1-\tilde{\theta}) \phi .
\end{aligned}
$$

Since $\sin x<0$ for $x \in(\pi, 2 \pi)$ and $\phi \geq 0$ in $R_{2},(2.10)$ follows from (2.7), (2.11) and (2.12).

The following theorem is as same as Theorem 5.1 in [4]. Here we give a different proof. 
THEOREM 2.3. If $\theta \leq \tilde{\theta}$, then $u \leq \tilde{u}$ in $\Omega$ and hence $u=0$ on $\partial \Omega$.

Proof. Recall that $u$ satisfies

$$
\int_{\Omega} h^{3} \nabla u \cdot \nabla(\phi-u) \geq \int_{\Omega_{1}} \gamma g(\phi-u)_{x}+\int_{\Omega_{2}} g(\phi-u)_{x}, \forall \phi \in K_{0} .
$$

Since $\phi=u \pm(u-\tilde{u})^{+} \in K_{0}, \gamma=1$ if $u>0$ and $(u-\tilde{u})^{+}=0$ if $u=0$, we get

$$
\int_{\Omega} h^{3} \nabla u \cdot \nabla(u-\tilde{u})^{+}=\int_{\Omega} g\left[(u-\tilde{u})^{+}\right]_{x} .
$$

Also, taking $\phi=\tilde{u}+(u-\tilde{u})^{+} \in K_{0}$ in (2.10),

$$
\int_{\Omega} h^{3} \nabla \tilde{u} \cdot \nabla(u-\tilde{u})^{+} \geq \int_{\Omega} \tilde{g}\left[(u-\tilde{u})^{+}\right]_{x} .
$$

Subtracting (2.15) from (2.14) we obtain

$$
\int_{\Omega} h^{3}\left|\nabla(u-\tilde{u})^{+}\right|^{2} \leq \int_{\Omega}(g-\tilde{g})\left[(u-\tilde{u})^{+}\right]_{x} .
$$

Since $g \geq \tilde{g}$ by assumption and $(g-\tilde{g})_{x}=0$, an integration by parts of the integral on the right-hand side of (2.16) gives

$$
\int_{\Omega}(g-\tilde{g})\left[(u-\tilde{u})^{+}\right]_{x}=-\int_{\partial_{0} \Omega}(g-\tilde{g}) u \leq 0 .
$$

Hence $(u-\tilde{u})^{+}=0$ and the theorem follows.

Remark 2.4. Note that $0<\tilde{\theta}<1$ was proved in [4]. However, (2.8) must be verified in order to ensure the existence of $\theta$ in the hypotheses of Theorem 2.3.

Next, we shall prove some properties of the free boundary $S$. Let

$$
K=\left\{\psi \in H^{1}\left(\Omega_{2}\right) ; \psi=u \quad \text { on } \quad T, \psi=0 \quad \text { on } \quad \partial \Omega_{2} \backslash T, \psi \geq 0 \quad \text { a.e. in } \quad \Omega_{2}\right\} .
$$

Notice that the set $K$ is a closed convex subset of $H^{1}\left(\Omega_{2}\right)$. By the matching lemma (cf. [6, p.31]), the function

$$
\phi=\left\{\begin{array}{lll}
u & \text { in } & \Omega_{1} \\
\psi & \text { in } & \Omega_{2}
\end{array}\right.
$$

is in $K_{0}$ for any $\psi \in K$. Hence, by $(0.8)$,

$$
\int_{\Omega_{2}} h^{3} \nabla u \cdot \nabla(\psi-u) \geq \int_{\Omega_{2}}(a \sin x)(\psi-u)
$$


for all $\psi \in K$. From general regularity results for variational inequalities( cf. [6, p.44] ) it follows that

$$
u \in C_{\text {loc }}^{1,1}\left(\Omega_{2}\right) .
$$

Lemma 2.5. Let $\Omega_{21}=\left\{(x, y) \in R_{1} ; g(x, y)<0\right\}=R_{1} \backslash \overline{\Omega_{1}}$. Then $u>0$ in $\Omega_{21} \cup\{x=$ $\pi\}$ and consequently $S$ is a union of two disjoint sets $S_{1}$ and $S_{2}$ such that $S_{1} \subset \Omega_{1} \cup T$ and $S_{2} \subset R_{2}$.

Proof. In $\Omega_{21}, u$ satisfies the inequality

$$
-\nabla \cdot\left(h^{3} \nabla u\right) \geq a \sin x>0 .
$$

From the non-negativity of $u$ and the strong maximum principle it follows that

$$
u>0 \text { in } \Omega_{21} \text {. }
$$

Hence $S$ is a union of two disjoint sets $S_{1}$ and $S_{2}$ such that $S_{1} \subset \Omega_{1} \cup T$ and $S_{2} \subset$ $R_{2} \cup\{x=\pi\}$. We claim that $S_{2} \subset R_{2}$. If there is a point $\left(x_{0}, y_{0}\right) \in S_{2}$ with $x_{0}=\pi$, then $u\left(x_{0}, y_{0}\right)=0$ and $\nabla u\left(x_{0}, y_{0}\right)=0$ by (2.17). On the other hand, by the Hopf boundary point lemma, we have

$$
\frac{\partial u}{\partial x}<0 \text { at }\left(x_{0}, y_{0}\right)
$$

which is a contradiction.

Proposition 2.6. If $\theta \leq \tilde{\theta}$ then $S_{2} \neq \emptyset$.

Proof. This is a simple consequence of Theorems 2.2 and 2.3 .

Proposition 2.7. If $\theta \leq \tilde{\theta}$ and $\theta$ is not identically equal to $\tilde{\theta}$, then $S_{1} \neq \emptyset$.

Proof. Suppose that $S_{1}=\emptyset$. Then $u>0$ in $\Omega_{1}$ and $\gamma \equiv 1$ in $\Omega_{1}$. For any $\phi \in \tilde{K} \subset K_{0}$ we have

$$
\int_{\Omega} h^{3} \nabla u \cdot \nabla(\phi-u) \geq \int_{\Omega_{1}} g(\phi-u)_{x}+\int_{\Omega_{2}}(a \sin x)(\phi-u) .
$$

Since

$$
\int_{\Omega_{1}} g(\phi-u)_{x}=\int_{\Omega_{1}}(a \sin x)(\phi-u)
$$

using $g=0$ on $T$ and $\phi=u=0$ on $\partial \Omega$, we conclude from (2.18) that $u$ is a solution of $\operatorname{Problem}(\tilde{P})$. Therefore, $u \equiv \tilde{u}$ by the uniqueness of solution to $\operatorname{Problem}(\tilde{P})$. Since

$$
\frac{\partial u}{\partial x}=\frac{1-\theta}{(1+a)^{2}} \quad \text { and } \quad \frac{\partial \tilde{u}}{\partial x}=\frac{1-\tilde{\theta}}{(1+a)^{2}} \quad \text { on } \quad \partial_{0} \Omega,
$$

we obtain that $\theta \equiv \tilde{\theta}$, a contradiction. 
Lemma 2.8. The set $\Omega_{2}^{+}$is connected.

Proof. Suppose that there is a component $G$ of $\Omega_{2}^{+}$such that $G \subset R_{2}$. Since

$$
\begin{aligned}
-\nabla \cdot\left(h^{3} \nabla u\right) & =a \sin x<0 \quad \text { in } \quad G, \\
u & =0 \text { on } \partial G,
\end{aligned}
$$

it follows from the maximum principle that $u<0$ in $G$, a contradiction.

Lemma 2.9. $\gamma_{x} \geq 0$ in $\Omega_{1}$ in the distribution sense.

Proof. The idea of proof is essentially due to Alt[1]. For any $\zeta \in C_{0}^{\infty}\left(\Omega_{1}\right)$, the function $\phi=u \pm \zeta$ is in $K_{0}$. Hence we obtain

$$
\int_{\Omega_{1}} h^{3} \nabla u \cdot \nabla \zeta=\int_{\Omega_{1}} \gamma g \zeta_{x}
$$

It follows that

$$
-\nabla \cdot\left(h^{3} \nabla u\right)+\gamma g_{x}+\gamma_{x} g=0 \text { in } \Omega_{1}
$$

in the distribution sense.

For any $\zeta \in C_{0}^{\infty}\left(\Omega_{1}\right), \zeta \geq 0$ and $\epsilon>0$, we have

$$
\int_{\Omega_{1}} h^{3} \nabla u \cdot \nabla[\min (u, \epsilon \zeta)]=\int_{\Omega_{1}} \gamma g[\min (u, \epsilon \zeta)]_{x}
$$

or

$$
\epsilon \int_{\Omega_{1} \cap\{u>\epsilon \zeta\}} h^{3} \nabla u \cdot \nabla \zeta+\int_{\Omega_{1} \cap\{u \leq \epsilon \zeta\}} h^{3}|\nabla u|^{2}=\int_{\Omega_{1}} g[\min (u, \epsilon \zeta)]_{x},
$$

since $u>0$ implies that $\gamma=1$. The right-hand side of (2.21) can be computed as

$$
\begin{aligned}
\int_{\Omega_{1}} g[\min (u, \epsilon \zeta)]_{x} & =-\int_{\Omega_{1}} g_{x}[\min (u, \epsilon \zeta)] \\
& =-\int_{\Omega_{1}^{+}} g_{x}[\min (u, \epsilon \zeta)] \\
& =-\epsilon \int_{\Omega_{1}^{+}} g_{x} \zeta+\int_{\Omega_{1}^{+}} g_{x}[\epsilon \zeta-\min (u, \epsilon \zeta)] \\
& =-\epsilon \int_{\Omega_{1}^{+}} \gamma g_{x} \zeta+\epsilon \int_{\Omega_{1}^{+}} g_{x}(\zeta-u / \epsilon)^{+} .
\end{aligned}
$$

Therefore, we obtain

$$
\int_{\Omega_{1} \cap\{u>\epsilon \zeta\}} h^{3} \nabla u \cdot \nabla \zeta+\int_{\Omega_{1}^{+}} \gamma g_{x} \zeta \leq \int_{\Omega_{1}^{+}} g_{x}(\zeta-u / \epsilon)^{+} .
$$


Using $g=0$ on $T$, the right-hand side of (2.25) is equal to

$$
\int_{B}(a \sin x) \zeta
$$

Hence we obtain from $(2.25)$

$$
\int_{T \cap B} h^{3}\left(\frac{\partial u^{l}}{\partial n}-\frac{\partial u^{r}}{\partial n}\right) \zeta \geq 0
$$

i.e.,

$$
\frac{\partial u^{l}}{\partial n}-\frac{\partial u^{r}}{\partial n} \geq 0 \text { on } T \cap B .
$$

This is a contradiction, since $\partial u^{l} / \partial n<0$ and $\partial u^{r} / \partial n>0$ at $P$ by the Hopf boundary point lemma. Hence $u(P)>0$.

Defining

$$
\begin{array}{rlrl}
\rho_{1}\left(y_{0}\right)=\inf \left\{x ; u\left(x, y_{0}\right)>0,\left(x, y_{0}\right) \in \Omega_{1}\right\} & \text { if } & \left(x_{0}, y_{0}\right) \in \Omega_{1}^{+} \text {for some } x_{0}, \\
=\rho\left(y_{0}\right) & \text { if } \quad u\left(x, y_{0}\right)=0, \forall\left(x, y_{0}\right) \in \Omega_{1},
\end{array}
$$

we conclude that (2.24) holds. Finally, the upper semi-continuity of $\rho_{1}$ follows from the continuity of $u$.

As a consequence of Lemmas 2.8 and 2.10 , we have:

ThEOREM 2.11. The set $\Omega^{+}$is connected and

$$
S_{1}=\left\{(x, y) ; x=\rho_{1}(y), y \in(0,1) \text { such that } \rho_{1}(y)>0\right\} .
$$

§3. Regularity of free boundary. In the rest of this paper we shall assume that

$$
\theta<\tilde{\theta}
$$

then by Theorem 2.3, Propositions 2.6 and $2.7 u=0$ on $\partial \Omega, S_{1} \neq \emptyset$ and $S_{2} \neq \emptyset$.

Lemma 3.1. Let $0<y_{1}<y_{2}<1$ and $x_{0}>0$ such that

$$
\begin{aligned}
& \text { (a) } x_{0} \leq \min _{y \in\left[y_{1}, y_{2}\right]} \rho(y), \\
& \text { (b) } u=0 \text { on }\left(0, x_{0}\right) \times\left\{y_{1}, y_{2}\right\}
\end{aligned}
$$

Then

$$
\int_{Q}\left(-h^{3} u_{x}+\gamma g\right) \leq 0
$$


Proof. We adopt a method from [5]. Introduce $Q \equiv\left(0, x_{0}\right) \times\left(y_{1}, y_{2}\right)$. Then $Q \subset \Omega_{1}$ by assumption (a). Let $\zeta \in H^{1}(Q) \cap C^{0}(\bar{Q})$ be a non-negative function such that $\zeta=0$ on $x=x_{0}$. Then for any $\epsilon>0$ the function

$$
\phi=\left\{\begin{array}{l}
u \pm \min (u, \epsilon \zeta) \text { in } Q \\
u \text { elsewhere }
\end{array}\right.
$$

is in $K_{0}$ by assumption (b). Hence

$$
\int_{Q} h^{3} \nabla u \cdot \nabla[\min (u, \epsilon \zeta)]=\int_{Q} \gamma g[\min (u, \epsilon \zeta)]_{x},
$$

or

$$
\epsilon \int_{Q \cap\{u>\epsilon \zeta\}} h^{3} \nabla u \cdot \nabla \zeta+\int_{Q \cap\{u \leq \epsilon \zeta\}} h^{3}|\nabla u|^{2}=\int_{Q} g[\min (u, \epsilon \zeta)]_{x}
$$

since $u>0$ implies that $\gamma=1$. Therefore, we obtain

$$
\int_{Q \cap\{u>\epsilon \zeta\}} h^{3} \nabla u \cdot \nabla \zeta-\int_{Q} g \zeta_{x} \leq-\int_{Q} g\left[(\zeta-u / \epsilon)^{+}\right]_{x} .
$$

The right-hand side of (3.2) can be computed as

$$
\begin{aligned}
-\int_{Q} g\left[(\zeta-u / \epsilon)^{+}\right]_{x} & =-\int_{y_{1}}^{y_{2}} \int_{0}^{x_{0}} g\left[(\zeta-u / \epsilon)^{+}\right]_{x} d x d y \\
& =\int_{y_{1}}^{y_{2}} \int_{0}^{x_{0}} g_{x}(\zeta-u / \epsilon)^{+} d x d y \\
& -\int_{y_{1}}^{y_{2}}\left[g(\zeta-u / \epsilon)^{+}\right]\left(x_{0}, y\right) d y+\int_{y_{1}}^{y_{2}}\left[g(\zeta-u / \epsilon)^{+}\right](0, y) d y \\
& \leq \int_{y_{1}}^{y_{2}} g(0, y) \zeta(0, y) d y
\end{aligned}
$$

using the facts that $\zeta=0$ on $x=x_{0}$ and $g_{x}<0$ in $Q$. Letting $\epsilon \rightarrow 0$ in (3.2), we obtain

$$
\int_{Q} h^{3} \nabla u \cdot \nabla \zeta-\int_{Q} g \zeta_{x} \leq \int_{y_{1}}^{y_{2}} g(0, y) \zeta(0, y) d y
$$

Now, let $\sigma(x)=x_{0}-x$ in $\left[0, x_{0}\right]$ and $=0$ for $x>x_{0}$. Define

$$
\eta_{\delta}(y)=\left\{\begin{array}{l}
1 \text { if } y \in\left[y_{1}+\delta, y_{2}-\delta\right] \\
0 \text { if } y \notin\left(y_{1}, y_{2}\right), \\
\text { linear if } y \in\left[y_{1}, y_{1}+\delta\right] \cup\left[y_{2}-\delta, y_{2}\right]
\end{array}\right.
$$


for any small positive constant $\delta$. Then the function

$$
\phi_{ \pm}^{\delta}(x, y)=u(x, y) \pm \sigma(x) \eta_{\delta}(y)
$$

is in $K_{0}$. Let

$$
\zeta^{\delta}(x, y)=\sigma(x)\left[1-\eta_{\delta}(y)\right]
$$

Then $\zeta^{\delta} \in H^{1}(Q) \cap C^{0}(\bar{Q})$ and satisfies

$$
\zeta^{\delta} \geq 0 \quad \text { in } \quad Q, \quad \zeta^{\delta}=0 \quad \text { on } \quad x=x_{0}
$$

Therefore, we obtain

$$
\begin{aligned}
\int_{Q}\left(-h^{3} u_{x}+\gamma g\right) & =\int_{Q}\left(h^{3} \nabla u \cdot \nabla \sigma-\gamma g \sigma_{x}\right) \\
& =\int_{Q}\left[h^{3} \nabla u \cdot \nabla\left(\sigma \eta_{\delta}\right)-\gamma g\left(\sigma \eta_{\delta}\right)_{x}\right] \\
& +\int_{Q}\left[h^{3} \nabla u \cdot \nabla\left[\sigma\left(1-\eta_{\delta}\right)\right]-\gamma g\left[\sigma\left(1-\eta_{\delta}\right)\right]_{x}\right]
\end{aligned}
$$

The first integral on the right-hand side of (3.4) vanishes since $\phi_{ \pm}^{\delta} \in K_{0}$. From (3.3) it follows that the second integral on the right-hand side of (3.4) is less than or equal to

$$
\int_{y_{1}}^{y_{2}} g(0, y) x_{0}\left[1-\eta_{\delta}(y)\right] d y+\int_{Q}(1-\gamma) g\left[\sigma\left(1-\eta_{\delta}\right)\right]_{x} .
$$

Since the second integral in (3.5) is less than or equal to zero and the first integral in (3.5) tends to zero as $\delta$ tends to zero, the lemma follows.

LEMMA 3.2. If $u=0$ in a ball $B=B_{r}\left(x_{0}, y_{0}\right)$ which is contained in $\Omega_{1}$ for some $r>0$, then $\gamma=0$ a.e. in $\tilde{B}$, where

$$
\tilde{B}=\left\{(x, y) ; x<x_{0},\left|y-y_{0}\right|<r\right\} \cup B
$$

Proof. For any $y_{1}<y_{2}$ with $y_{1} \geq y_{0}-r$ and $y_{2} \leq y_{0}+r$, by Lemmas 2.10 and 3.1, we have

$$
\int_{Q}\left(-h^{3} u_{x}+\gamma g\right) \leq 0
$$

where $Q=\left(0, x_{0}+\bar{x}\right) \times\left(y_{1}, y_{2}\right) \subset \tilde{B}$ and $\bar{x}=\min \left(\sqrt{r^{2}-\left(y_{2}-y_{0}\right)^{2}}, \sqrt{r^{2}-\left(y_{1}-y_{0}\right)^{2}}\right)$. Since $u=0$ in $\tilde{B}$ by Lemma $2.10,(3.6)$ implies that

$$
\int_{Q} \gamma g \leq 0
$$


From the facts $g>0$ and $\gamma \geq 0$ in $\Omega_{1}$ it follows that $\gamma=0$ a.e. in $Q$. Therefore, $\gamma=0$ a.e. in $\tilde{B}$.

Let $\delta$ be any positive constant. For any set $A$, let

$$
A^{*}=A \cup E, \quad \text { where } E=(-\delta, 0] \times(0,1) .
$$

Extend $u$ and $\gamma$ to be zero in $E$. Notice that $u \in H_{0}^{1}\left(\Omega^{*}\right)$ by Theorem 2.3. For any $\zeta \in C_{0}^{\infty}\left(\Omega_{1}^{*}\right)$ the function $\phi=u \pm \zeta$ restricted to $\Omega$ is in $K_{0}$. Hence

$$
\int_{\Omega_{1}} h^{3} \nabla u \cdot \nabla \zeta=\int_{\Omega_{1}} \gamma g \zeta_{x}
$$

Therefore, we obtain

$$
\int_{\Omega_{1}^{*}} h^{3} \nabla u \cdot \nabla \zeta=\int_{\Omega_{1}^{*}} \gamma g \zeta_{x}
$$

i.e., $u$ satisfies the equation

$$
-\nabla \cdot\left(h^{3} \nabla u\right)+(\gamma g)_{x}=0 \text { in } \Omega_{1}^{*}
$$

in the weak sense. Now, we are ready to prove the Lipschitz continuity of $S_{1} \backslash T$.

Theorem 3.3. The free boundary $S_{1} \backslash T$ is locally Lipschitz continuous.

Proof. Let $P \in S_{1} \backslash T$ and let $r_{0}$ be a positive number such that the ball $B_{0}=B_{r_{0}}(P)$ is contained in $\Omega_{1}$. Adapting a method of Alt[2], we introduce the function

$$
w(x, y)=\int_{-\delta}^{x} h^{3}(\xi) u(\xi, y) d \xi
$$

Then $u(x, y)=0$ if and only if $w(x, y)=0$. We have

$$
\begin{aligned}
w_{x} & =h^{3} u \\
w_{x x} & =h^{3} u_{x}+3 \frac{h^{\prime}}{h} w_{x}, \\
w_{y}(x, y) & =\int_{-\delta}^{x} h^{3}(\xi) u_{y}(\xi, y) d \xi .
\end{aligned}
$$

We approximate $\gamma$ by a sequence $\left\{\gamma_{n}\right\}$ of functions in $C^{\infty}\left(\Omega_{1}^{*}\right) \cap L^{\infty}\left(\Omega_{1}^{*}\right)$ such that $\gamma_{n}=0$ in a neighborhood of $\{x=-\delta, 0<y<1\}$. For each $n$ let $u_{n}$ be the solution of

$$
\left\{\begin{array}{l}
-\nabla \cdot\left(h^{3} \nabla u_{n}\right)+\left(\gamma_{n} g\right)_{x}=0 \text { in } \Omega_{1}^{*} \\
u_{n}=u \text { on } \partial \Omega_{1}^{*} .
\end{array}\right.
$$


Note that $u_{n} \rightarrow u$ uniformly in $\overline{\Omega_{1}^{*}}$ and strongly in $H^{1}\left(\Omega_{1}^{*}\right)$. Let $w_{n}$ be defined as in (3.8) for $u$ being replaced by $u_{n}$. We compute using (3.9) and $u_{n, x}(-\delta, y)=\gamma_{n}(-\delta, y)=0$

$$
\begin{aligned}
w_{n, y y}(x, y) & =\int_{-\delta}^{x}\left[h^{3}(\xi) u_{n, y}(\xi, y)\right]_{y} d \xi \\
& =-\int_{-\delta}^{x}\left[h^{3}(\xi) u_{n, \xi}(\xi, y)\right]_{\xi} d \xi+\int_{-\delta}^{x}\left(\gamma_{n} g\right)_{\xi}(\xi, y) d \xi \\
& =-w_{n, x x}(x, y)+3\left(\frac{h^{\prime}}{h} w_{n, x}\right)(x, y)+\left(\gamma_{n} g\right)(x, y) .
\end{aligned}
$$

Hence we obtain

$$
-\Delta w_{n}+3 \frac{h^{\prime}}{h} w_{n, x}=-\gamma_{n} g \quad \text { in } \quad \Omega_{1}^{*}
$$

Therefore, for any $\zeta \in C_{0}^{\infty}\left(\Omega_{1}^{*}\right)$ we have

$$
\int_{\Omega_{1}^{*}} \nabla w_{n} \cdot \nabla \zeta+\int_{\Omega_{1}^{*}} 3 \frac{h^{\prime}}{h} w_{n, x} \zeta+\int_{\Omega_{1}^{*}} \gamma_{n} g \zeta=0 .
$$

Letting $n \rightarrow \infty$ in (3.11), we obtain that $w$ satisfies

$$
-\Delta w+3 \frac{h^{\prime}}{h} w_{x}=-\gamma g \text { in } \Omega_{1}^{*}
$$

in the weak sense. Since $\gamma \in L^{\infty}\left(\Omega_{1}^{*}\right)$, (3.12) holds in the strong sense. Recall that $0 \leq \gamma \leq 1$ and $\gamma=1$ if $w>0$. Hence $w$ satisfies the variational inequality

$$
\begin{aligned}
-\Delta w+3 \frac{h^{\prime}}{h} w_{x} & \geq-g, \\
w & \geq 0, \\
w\left[-\Delta w+3 \frac{h^{\prime}}{h} w_{x}+g\right] & =0
\end{aligned}
$$

a.e. in $\Omega_{1}$ and $w_{x}>0$ in $\Omega_{1}^{+}$. Note that $w \in C_{\text {loc }}^{1,1}\left(\Omega_{1}\right)$ by general regularity results for variational inequalities (cf. [6, p.44]).

For any $r \in\left(0, r_{0}\right)$, let $B_{r}=B_{r}(P)$ and $B_{r / 2}=B_{r / 2}(P)$. Let $\zeta$ be a smooth function such that $0 \leq \zeta \leq 1, \zeta=1$ on $\partial B_{r}$ and $\zeta=0$ in $B_{r / 2}$. Following the method used in [8], we consider the function

$$
v(x, y)=-b w_{x}(x, y)+c w_{y}(x, y)+w(x, y)-d \zeta(x, y)
$$

where $b, c$ and $d$ are constants with $b>0,|c|$ small and $d>0$. Since

$$
\begin{aligned}
-\Delta w & =-3 \frac{h^{\prime}}{h} w_{x}-g \\
\Delta w_{x} & =3 \frac{h^{\prime}}{h} w_{x x}+3 \frac{h^{\prime \prime}}{h} w_{x}-3 \frac{h^{\prime 2}}{h^{2}} w_{x}-a \sin x \\
-\Delta w_{y} & =-3 \frac{h^{\prime}}{h} w_{x y}+(1+a) \theta^{\prime}
\end{aligned}
$$


in $B_{r} \cap \Omega_{1}^{+}$, we obtain

$$
\begin{aligned}
-\Delta v+3 \frac{h^{\prime}}{h} v_{x} & =3 b w_{x}\left(\frac{h^{\prime \prime}}{h}-\frac{h^{\prime 2}}{h^{2}}\right)-b a \sin x \\
& +c(1+a) \theta^{\prime}-g+d\left(\Delta \zeta-3 \frac{h^{\prime}}{h} \zeta_{x}\right)
\end{aligned}
$$

in $B_{r} \cap \Omega_{1}^{+}$. Since $a \sin x \geq \delta$ in $B_{0}$ for some positive constant $\delta$ and there is a positive constant $M$ such that

$$
\|w\|_{C^{1,1}\left(B_{0}\right)} \leq M
$$

one can choose $r$ so small that the first two terms on the right-hand side of (3.14) is less than or equal to $-b \delta / 2$. Hence we obtain

$$
-\Delta v+3 \frac{h^{\prime}}{h} v_{x} \leq 0 \quad \text { in } \quad B_{r} \cap \Omega_{1}^{+}
$$

if $b$ is sufficiently large and $|c|$ and $d$ are sufficiently small. For the boundary conditions, we have

$$
\begin{aligned}
& v=-d \zeta \leq 0 \quad \text { on } \quad S_{1} \\
& v \leq c w_{y}+d / 2-d \leq 0 \quad \text { on } \quad \partial B_{r} \cap \Omega_{1}^{+} \cap\{w \leq d / 2\}
\end{aligned}
$$

if $|c|$ is sufficiently small, and

$$
v \leq-b \inf _{\Gamma} w_{x}+d / 2+\sup w-d \leq 0 \quad \text { on } \quad \Gamma \equiv \partial B_{r} \cap \Omega_{1}^{+} \cap\{w>d / 2\}
$$

if $b$ is sufficiently large. Therefore, it follows from (3.15) and the maximum principle that $v \leq 0$ in $B_{r} \cap \Omega_{1}^{+}$. In particular, we have

$$
-b w_{x}+c w_{y} \leq 0 \text { in } B_{r / 2}
$$

and the theorem follows.

Remark 3.4. From (3.16) and the regularity theorems for the free boundary of variational inequality(cf. [6, Chap. 2]) it follows that $S_{1} \backslash T$ is in $C_{\text {loc }}^{m+1, \alpha}$ if $g \in C^{m, \alpha}$, where $m \geq 1$ and $0<\alpha<1$. Notice that the inhomogeneous term $-g$ in (3.13) being strictly uniformly negative is needed to get $C^{1}$ regularity.

Remark 3.5. The proof of Theorem 3.3 cannot be applied at the points $P \in S_{1} \cap T$, since we do not know whether $u$ satisfies an elliptic equation in a neighborhood of $P$.

In the sequel, we let $\pi_{y}(A)$ be the projection of $A$ onto the y-axis. 
TheOREM 3.6. $\rho_{1}(y)$ is continuous.

Proof. In view of Theorem 3.3, it remains to prove the continuity of $\rho_{1}$ only at points $y \in \pi_{y}\left(S_{1} \cap T\right)$. Suppose for contradiction that $\rho_{1}$ is not continuous at $y_{0}$ for some $y_{0} \in \pi_{y}\left(S_{1} \cap T\right)$. Since $\rho_{1}$ is upper semi-continuous, there is a sequence $y_{n} \rightarrow y_{0}$ such that

$$
x_{0} \equiv \lim _{n \rightarrow \infty} \rho_{1}\left(y_{n}\right)<\rho_{1}\left(y_{0}\right)=\rho\left(y_{0}\right) .
$$

Set $x_{n}=\rho_{1}\left(y_{n}\right), P_{0}=\left(x_{0}, y_{0}\right)$ and $P_{n}=\left(x_{n}, y_{n}\right)$. Let $r_{0}$ be a positive number such that $B=B_{r_{0}}\left(P_{0}\right) \subset \Omega_{1}$. Then there are positive constants $\delta$ and $M$ such that

$$
a \sin x \geq \delta \quad \text { in } \quad B, \quad\|w\|_{C^{1,1}(B)} \leq M .
$$

Without loss of generality we may assume that $P_{n} \in B_{r_{0} / 2}\left(P_{0}\right), \forall n \geq 1$. From the proof of Theorem 3.3 there exist positive constants $\omega$ and $r$ depending only on $\delta$ and $M$ such that $u>0$ in $A_{\omega}\left(P_{n}\right) \cap B_{r}\left(P_{n}\right)$ for all $n$, where $A_{\omega}\left(P_{n}\right)$ is the cone with axis parallel to x-axis, vertex at $P_{n}$ and opening angle $\omega$. This is a contradiction, since $u=0$ on $\left\{0 \leq x \leq \rho\left(y_{0}\right), y=y_{0}\right\}$ by Lemma 2.10 . Hence the theorem follows.

Corollary 3.7. For any solution $(u, \gamma)$ of $\operatorname{Problem}\left(P_{0}\right)$,

$$
\gamma=I_{\Omega_{1}^{+}} \text {a.e. in } \Omega_{1} \text {. }
$$

Proof. Let $\left(x_{0}, y_{0}\right)$ be a point in $\Omega_{1}$ with $x_{0}<\rho_{1}\left(y_{0}\right)$. From Theorem 3.6 it follows that there exists a positive number $r$ such that the ball $B=B_{r}\left(x_{0}, y_{0}\right)$ is contained in the set $\{u=0\}$. By Lemma 3.2, we obtain that $\gamma=0$ a.e. in $\tilde{B}$, where $\tilde{B}$ is defined as in Lemma 3.2. Since the point $\left(x_{0}, y_{0}\right)$ is arbitrary and the measure of the set $\{(x, y) ; x=$ $\left.\rho_{1}(y)\right\}$ is zero, the corollary follows.

Proposition 3.8. The set $\left\{y ; \rho_{1}(y)=\rho(y)\right\}$ has no interior points.

Proof. Suppose that there exist $y_{0}$ and $\delta>0$ such that

$$
\rho_{1}(y)=\rho(y), \forall y \in\left(y_{0}-\delta, y_{0}+\delta\right) .
$$

Let $P=\left(\rho\left(y_{0}\right), y_{0}\right)$ and $B=B_{\delta}(P)$. Recall the notations $n, u^{r}$ and $u^{l}$ in the proof of Lemma 2.10. Then by the Hopf boundary lemma we have

$$
\frac{\partial u^{r}}{\partial n}>0 \text { on } B \cap T .
$$

On the other hand, for any $\zeta \in C_{0}^{\infty}(B)$ with $\zeta \geq 0$, since $u=\gamma=0$ in $B \cap \Omega_{1}$, we have

$$
\int_{B \cap \Omega_{2}} h^{3} \nabla u \cdot \nabla \zeta \geq \int_{B \cap \Omega_{2}}(a \sin x) \zeta .
$$

The left-hand side of (3.17) can be computed as

$$
\int_{B \cap \Omega_{2}} h^{3} \nabla u \cdot \nabla \zeta=\int_{B \cap \Omega_{2}}(a \sin x) \zeta-\int_{B \cap T} h^{3} \frac{\partial u^{r}}{\partial n} \zeta .
$$

Hence we obtain from (3.17) that $\partial u^{r} / \partial n \leq 0$ on $B \cap T$, a contradiction. 
Proposition 3.9. The closure of $\pi_{y}\left(S_{1}\right)$ is $[0,1]$.

Proof. Suppose that there is $y_{0}$ such that $y_{0} \in(0,1) \backslash A$, where $A=\overline{\pi_{y}\left(S_{1}\right)}$. Then there exists a positive constant $\delta$ such that $J \equiv\left(y_{0}-\delta, y_{0}+\delta\right) \subset(0,1) \backslash A$. Hence we have $\rho_{1}(y)=0, \forall y \in J$. Let $B=B_{\delta}\left(0, y_{0}\right)$. For any $\zeta \in C_{0}^{\infty}(B)$, since the function $u \pm \zeta$ restricted to $\Omega$ is in $K_{0}$ and $u>0$ in $B \cap \Omega$, we have

$$
\int_{B \cap \Omega_{1}} h^{3} \nabla u \cdot \nabla \zeta=\int_{B \cap \Omega_{1}} g \zeta_{x}
$$

or

$$
\int_{B \cap \Omega_{1}}(a \sin x) \zeta-\int_{B \cap \partial_{0} \Omega} h^{3} u_{x} \zeta=\int_{B \cap \Omega_{1}}(a \sin x) \zeta-\int_{B \cap \partial_{0} \Omega} g \zeta .
$$

Hence

$$
u_{x}=\frac{1-\theta}{(1+a)^{2}} \quad \text { on } \quad B \cap \partial_{0} \Omega .
$$

From (2.7), (3.18) and Theorem 2.3 it follows that

$$
\frac{1-\theta(y)}{(1+a)^{2}} \leq \frac{1-\tilde{\theta}(y)}{(1+a)^{2}}, \forall y \in J
$$

That is we have $\theta(y) \geq \tilde{\theta}(y)$ for all $y \in J$. This contradicts to the assumption that $\theta<\tilde{\theta}$ in $(0,1)$. Therefore, the set $(0,1) \backslash A$ is empty. Since $A$ is closed, the proposition follows.

Remark 3.10. If one can show that $S_{1} \cap T=\emptyset$ and $S_{2}$ is smooth, then by Theorem 3.3 $u$ will be a classical solution of $\operatorname{Problem}\left(P_{c}\right)$ for any solution $(u, \gamma)$ of $\operatorname{Problem}\left(P_{0}\right)$. Note that if $S_{1} \cap T \neq \emptyset$ then $S_{1}$ cannot be smooth near any point $P \in S_{1} \cap T$. Indeed, otherwise we have $\partial u / \partial \nu<0$ at $P$ by the Hopf boundary point lemma. But from (0.5) and the fact $g=0$ on $T$ it follows that $\partial u / \partial \nu=0$ at $P$, a contradiction. Note also that if one can prove that $S_{2}$ is a y-graph, then $S_{2}$ will be locally real analytic by the same reasoning as in Theorem 2.2 .

$\S 4$. Uniqueness. Let $\left(u_{1}, \gamma_{1}\right)$ and $\left(u_{2}, \gamma_{2}\right)$ be two solutions of $\operatorname{Problem}\left(P_{0}\right)$. We shall prove that $u_{1}=u_{2}$ and $\gamma_{1}=\gamma_{2}$ using the method of Carrillo and Chipot(cf. [5]). Let $\rho_{i}$ be the function defined in Lemma 2.10 corresponding to the solution $\left(u_{i}, \gamma_{i}\right), i=1,2$, and let

$$
\begin{aligned}
u_{0} & =\min \left(u_{1}, u_{2}\right), \quad \gamma_{0}=\min \left(\gamma_{1}, \gamma_{2}\right), \quad \rho_{0}=\min \left(\rho_{1}, \rho_{2}\right), \\
D_{i} & =\left\{y \in(0,1) ; \rho_{i}(y)>\rho_{0}(y)\right\}, i=1,2 .
\end{aligned}
$$

Lemma 4.1. For any $\zeta \in H^{1}(\Omega) \cap C^{0}(\bar{\Omega})$ such that $\zeta \geq 0$ in $\Omega$, we have

$$
\begin{aligned}
& \int_{\Omega} h^{3} \nabla\left(u_{1}-u_{0}\right) \cdot \nabla \zeta-\int_{\Omega_{1}}\left(\gamma_{1}-\gamma_{0}\right) g \zeta_{x} \leq \int_{D_{2}}(g \zeta)\left(\rho_{1}(y), y\right) d y, \\
& \int_{\Omega} h^{3} \nabla\left(u_{2}-u_{0}\right) \cdot \nabla \zeta-\int_{\Omega_{1}}\left(\gamma_{2}-\gamma_{0}\right) g \zeta_{x} \leq \int_{D_{1}}(g \zeta)\left(\rho_{2}(y), y\right) d y .
\end{aligned}
$$


Proof. Let $\epsilon$ be an arbitrary small positive number. Since $\phi=u_{1} \pm \min \left(u_{1}-u_{0}, \epsilon \zeta\right) \epsilon$ $K_{0}$, we have

$$
\begin{aligned}
& \int_{\Omega} h^{3} \nabla u_{1} \cdot \nabla {\left[\min \left(u_{1}-u_{0}, \epsilon \zeta\right)\right] } \\
&=\int_{\Omega_{1}} \gamma_{1} g\left[\min \left(u_{1}-u_{0}, \epsilon \zeta\right)\right]_{x}+\int_{\Omega_{2}}(a \sin x)\left[\min \left(u_{1}-u_{0}, \epsilon \zeta\right)\right] .
\end{aligned}
$$

On the other hand, $\phi=u_{2}+\min \left(u_{1}-u_{0}, \epsilon \zeta\right) \in K_{0}$ implies that

$$
\begin{aligned}
& \int_{\Omega} h^{3} \nabla u_{2} \cdot \nabla {\left[\min \left(u_{1}-u_{0}, \epsilon \zeta\right)\right] } \\
& \geq \int_{\Omega_{1}} \gamma_{2} g\left[\min \left(u_{1}-u_{0}, \epsilon \zeta\right)\right]_{x}+\int_{\Omega_{2}}(a \sin x)\left[\min \left(u_{1}-u_{0}, \epsilon \zeta\right)\right]
\end{aligned}
$$

Substracting (4.4) from (4.3), we get

$$
\int_{\Omega} h^{3} \nabla\left(u_{1}-u_{2}\right) \cdot \nabla\left[\min \left(u_{1}-u_{0}, \epsilon \zeta\right)\right] \leq \int_{\Omega_{1}}\left(\gamma_{1}-\gamma_{2}\right) g\left[\min \left(u_{1}-u_{0}, \epsilon \zeta\right)\right]_{x} .
$$

We write $u_{1}-u_{2}=\left(u_{1}-u_{0}\right)+\left(u_{0}-u_{2}\right)$ and $\gamma_{1}-\gamma_{2}=\left(\gamma_{1}-\gamma_{0}\right)+\left(\gamma_{0}-\gamma_{2}\right)$. Then from (4.5) and the identities $\left(u_{0}-u_{2}\right)\left(u_{1}-u_{0}\right)=0$ and $\left(\gamma_{0}-\gamma_{2}\right)\left(u_{1}-u_{0}\right)=0$ it follows that

$$
\int_{\Omega} h^{3} \nabla\left(u_{1}-u_{0}\right) \cdot \nabla\left[\min \left(u_{1}-u_{0}, \epsilon \zeta\right)\right] \leq \int_{\Omega_{1}}\left(\gamma_{1}-\gamma_{0}\right) g\left[\min \left(u_{1}-u_{0}, \epsilon \zeta\right)\right]_{x} .
$$

Since

$$
\int_{\Omega_{1}}\left(\gamma_{1}-\gamma_{0}\right) g\left[\min \left(u_{1}-u_{0}, \epsilon \zeta\right)\right]_{x}=\epsilon \int_{\Omega_{1}}\left(\gamma_{1}-\gamma_{0}\right) g \zeta_{x}-\epsilon \int_{\Omega_{1}}\left(\gamma_{1}-\gamma_{0}\right) g\left[\left(\zeta-\frac{u_{1}-u_{0}}{\epsilon}\right)^{+}\right]_{x}
$$

and

$$
\begin{aligned}
& \int_{\Omega} h^{3} \nabla\left(u_{1}-u_{0}\right) \cdot \nabla\left[\min \left(u_{1}-u_{0}, \epsilon \zeta\right)\right] \\
& =\epsilon \int_{\Omega \cap\left\{u_{1}-u_{0}>\epsilon \zeta\right\}} h^{3} \nabla\left(u_{1}-u_{0}\right) \cdot \nabla \zeta+\int_{\Omega \cap\left\{u_{1}-u_{0} \leq \epsilon \zeta\right\}} h^{3}\left|\nabla\left(u_{1}-u_{0}\right)\right|^{2},
\end{aligned}
$$

we obtain from (4.6)

$$
\int_{\Omega \cap\left\{u_{1}-u_{0}>\epsilon \zeta\right\}} h^{3} \nabla\left(u_{1}-u_{0}\right) \cdot \nabla \zeta \leq \int_{\Omega_{1}}\left(\gamma_{1}-\gamma_{0}\right) g \zeta_{x}-\int_{\Omega_{1}}\left(\gamma_{1}-\gamma_{0}\right) g\left[\left(\zeta-\frac{u_{1}-u_{0}}{\epsilon}\right)^{+}\right]_{x} .
$$


Wilhelm I. Fushchich, Exact Solutions of Multidimensional Nonlinear Dirac's and Schrödinger's Equations

Wilhelm I. Fushchich and Renat Zhdanov, On Some New Exact Solutions of Nonlineas D'Allembert and Hamilton Equations

Brian A. Coomes, The Lorenz System Does Not Have a Polynornial Flow

J.W. Helton and N.J. Young, Approximation of Hankel Operators: Truncation Error in an $H^{\infty}$ Design Method

Gregory Ammar and Paul Gader, A Variant of the Gohberg-Semencul Formula Involving Circulant Matrices

R.L. Fosdick and G.P. MacSithigh, Minimization in Nonlinear Elasticity Theory for Bodies Reinforced with Inextensible Cords

Fernando Reitich, Rapidly Stretching Plastic Jets: The Linearized Problem

Francisco Bernis and Avner Friedman, Higher Order Nonlineax Degenerate Parabolic Equations

Xinfu Chen and Avner Friedman, Maxwell's Equations in a Periodic Structure

Avner Friedman and Michael Vogelius Determining Cracks by Boundary Measurements

Yuji Kodama and John Gibbons, A Method for Solving the Dispersionless KP Hierarchy and its Exact Solutions II

Yuji Kodama, Exact Solutions of Hydrodynamic Type Equations Faving Infinitely Many Conserved Densities

Robert Carroll, Some Forced Nonlinear Equations and the Time Evolution of Spectral Data

Chjan. C. Lim Spanning Binary Trees, Symplectic Matrices, and Canonical Transformations for Classical N-body Problems

E.F. Assmus, Jr. and J.D. Key, Translation Planes and Derivation Sets

\section{Matthew Witten, Mathematical Modeling and Computer Simulation of the Aging-Cancer} Interface

Matthew Witten and Caleb E. Finch, Re-Examining The Gompertzian Model of Aging

Bei Hu, A Free Boundary Problem for a Hamilton-Jacobi Equation Arising in Ions Etching

T.C. Hu, Victor Klee and David Larman, Optimization of Globally Convex Functions

Pierre Goossens, Shellings of Tilings

D. David, D. D. Holm, and M.V. Tratnik, Integrable and Chaotic Polarization Dynamics in Nonlinear Optical Beams

D. David, D.D. Holm and M.V. Tratnik, Horseshoe Chaos in a Periodically Perturbed Polarized Optical Beam

Laurent Habsieger, Linear Recurrent Sequences and Irrationality Measures

Laurent Habsieger, MacDonald Conjectures and The Selberg Integral

David Kinderlehrer and Giorgio Vergara-Caffarelli, The Relaxation of Functionals with Surface Energies

Richard James and David Kinderlehrer, Theory of Diffusionless Phase Transitions

David Kinderlehrer, Recent Developments in Liquid Crystal Theory

Niky Kamran and Peter J. Olver, Equivalence of Higher Order Lagrangians

1. Formulation and Reduction
Lucas Hsu, Niky Kamran and Peter J. Olver, Equivalence of Higher Order Lagrangians II. The Cartan Form for Particle Lagrangians

D.J. Kaup and Peter J. Olver, Quantization of BiHamiltonian Systems

Metin Arik, Fahrünisa Neyzi, Yavuz Nutku, Peter J. Olver and John M. Verosky Multi-Hamiltonian Structure of the Born-Infeld Equation

David H. Wagner, Detonation Waves and Deflagration Waves in the One Dimensional ZND Model for High Mach Number Combustion

Jerrold R. Griggs and Daniel J. Kleitman, Minimum Cutsets for an Element of a Boolean Lattice

Dieter Jungnickel, On Affine Difference Sets

Pierre Leroux, Reduced Matrices and q-log Concavity Properties of q-Stirling Numbers

A. Narain and Y. Kizilyalli, The Flow of Pure Vapor Undergoing Film Condensation Between Parallel Plates

Donald A. French, On the Convergence of Finite Element Approximations of a Relaxed Variational Problem

Yisong Yang, Computation, Dimensionality, and Zero Dissipation Limit of the Ginzburg-Landau Wave Equation 
Title

506 Yisong Yang, A Note On Nonabelian Vortices

Chjan. C. Lim, Existence of Kam Tori in the Phase Space of Vortex Systems

John Weiss, Bäcklund Transformations and the Painlevé Property

Pu Fu-cho and D.H. Sattinger, The Yang-Baxter Equation for Integrable Systems

E. Bruce Pitman and David G. Schaeffer, Instability and Ill-Posedness in Granular Flow

Brian A. Coomes, Polynomial Flows on $\mathbf{C}^{n *}$

Bernardo Cockburn, Suchung Hou and Chi-Wang Shu, The Runge-Kutta Local

Projection Discontinuous Galerkin Finite Element Method for Conservation Laws IV:

The Multidimensional Case

Peter J. Olver, Invariant Theory, Equivalence Problems, and the Calculus of Variations

Daniel D. Joseph and Thomas S. Lundgren with an appendix by $R$. Jackson and D.A. Saville, Ensemble Averaged and Mixture Theory Equations.

P. Singh, Ph. Caussignac, A. Fortes, D.D. Joseph and T. Lundgren, Stability of Periodic Arrays of Cylinders Across the Stream by Direct Simulation

Daniel D. Joseph, Generalization of the Foscolo-Gibilaro Analysis of Dynamic Waves

A. Narain and D.D. Joseph, Note on the Balance of Energy at a Phase Change Interface

Daniel D. Joseph, Remarks on inertial radii, persistent normal stresses, secondary motions, and non-elastic extensional viscosities

D. D. Joseph, Mathematical Problems Associated with the Elasticity of Liquids

Henry C. Simpson and Scott J. Spector, Some Necessary Conditions at an Internal Boundary for Minimizers in Finite Elasticity

Peter Gritzmann and Victor Klee, On the 0-1 Maximization of Positive Definite Quadratic Forms

Fu-Cho Pu and D.H. Sattinger, The Yang-Baxter Equations and Differential Identities

Avner Friedman and Fernando Reitich, A Hyperbolic Inverse Problem Arising in the Evolution of Combustion Aerosol

E.G. Kalnins, Raphael D. Levine and Willard Miller, Jr., Conformal Symmetries and Generalized Recurrences for Heat and Schrödinger Equations in One Spatial Dimension

Wang Jinghua and Gerald Warnecke, On Entropy Consistency of Large Time Step Godunov and Glimm Schemes

C. Guillopé and J.C. Saut, Existence Results for the Flow of Viscoelastic Fluids with a Differential Constitutive Law

H.L. Bodlaender, P. Gritzmann, V. Klee and J. Van Leeuwen Computational Complexity of Norm-Maximization

Li Ta-tsien (Li Da-qian) and Yu Xin, Life-Span of Classical Solutions to Fully Nonlinear Wave Equations

Jong-Shenq Guo, A Variational Inequality Associated with a Lubrication Problem

Jong-Shenq Guo, On the Semilinear Elliptic Equation $\Delta w-\frac{1}{2} y \cdot \nabla w+\lambda w-w^{-\beta}=0$ in $R^{n}$

Andrew E. Yagle, Inversion of the Bloch transform in magnetic resonance imaging using asymmetric two-component inverse scattering

Bei Hu, A Fiber Tapering Problem

Peter J. Olver, Canonical Variables for BiHamiltonian Systems

Michael Renardy, A Well-Posed Boundary Value Problem for Supercritical Flow of Viscoelastic Fluids of Maxwell Type

Michael Renardy, Ill-Posedness Resulting from Slip As a Possible Explanation of Melt Fracture

Michael Renardy, Compatibility Conditions at Corners Between Walls and Inflow Boundaries for Fluids of Maxwell Type

Rolf Rees, The Spectrum of Restricted Resolvable Designs with $r=2$

D. Lewis and J.C. Simo, Nonlinear stability of rotating pseudo-rigid bodies

Robert Hardt and David Kinderlehrer, Variational Principles with Linear Growth

San Yih Lin and Yisong Yang, Computation of Superconductivity in Thin Films

A. Narain, Pressure Driven Flow of Pure Vapor Undergoing Laminar Film Condensation Between Parallel Plates

P.J. Vassiliou, On Local Equivalence for Vector Field Systems

Brian A. Coomes, On Conditions Sufficient for Injectivity of Maps

Yanchun Zhao, A Class of Global Smooth Solutions of the One Dimensional Gas Dynamics System

H. Holden, L. Holden and N.H. Risebro, Some Qualitative Properties of $2 \times 2$ Systems of Conservation Laws of Mixed Type

M. Slemrod, Dynamics of Measured Valued Solutions to a Backward-Forward Heat Equation 